\title{
Effects of Extracorporeal Shock Waves on Microcirculation and Angiogenesis in the in vivo Wound Model of the Diver Box
}

\author{
Heiko Sorg ${ }^{a, b}$ Inga Zwetzich ${ }^{c}$ Daniel Johannes Tilkorn ${ }^{d}$ \\ Jonas Kolbenschlag ${ }^{e}$ Jörg Hauser ${ }^{d}$ Ole Goertz ${ }^{f} \quad$ Nick Spindler $^{g}$ \\ Stefan Langerg Andrej Ring ${ }^{c}$ \\ aDepartment of Plastic, Reconstructive and Aesthetic Surgery, Klinikum Westfalen, Dortmund, Germany; \\ ${ }^{b}$ Department of Health, University of Witten/Herdecke, Witten, Germany; ${ }^{\mathrm{C} D e p a r t m e n t ~ o f ~ P l a s t i c, ~ R e c o n s t r u c t i v e ~}$ \\ and Aesthetic Surgery, Hand Surgery, St. Rochus Hospital Castrop-Rauxel, Castrop-Rauxel, Germany; ${ }^{\mathrm{d} D e p a r t m e n t}$ \\ of Plastic, Reconstructive and Aesthetic Surgery, Hand Surgery, Alfried Krupp Krankenhaus Essen, Essen, Germany; \\ eDepartment of Hand-, Plastic, Reconstructive and Burn Surgery, BG University Hospital Tübingen, Tübingen, \\ Germany; 'Department of Plastic, Reconstructive and Aesthetic Surgery, Hand Surgery, Martin-Luther-Krankenhaus, \\ Berlin, Germany; ${ }^{9}$ Department of Orthopedic Surgery, Traumatology and Plastic Surgery, University Hospital \\ Leipzig, Leipzig, Germany
}

\section{Keywords}

Diver Box · Dorsal skin fold chamber $\cdot$ Pulse rate $\cdot$ Shock wave $\cdot$ Angiogenesis $\cdot$ Wound healing

\begin{abstract}
Introduction: Extracorporeal shock waves (ESWs) have been shown to have a positive effect on skin wound healing; however, little is known on the regeneration of the microcirculation and angiogenesis as well as the different application modes. Methods: A total of $40 \mathrm{BALB} / \mathrm{C}$ mice were provided with dorsal skin fold chambers and were divided into 3 therapy groups $(n=30)$ and one control group $(n=10)$. The 3 therapy groups were treated with shock waves at different pulse rates (500-1,000 pulses/min) and application frequencies (day 0 and day 6 or day 0 only). Photographic documentation and intravital microscopy were carried out on day 1,2, 4, and 6 after wounding. Results: Using the newly developed Diver Box, shock waves could be applied in vivo without mechanical tissue damage. Shock wave therapy to skin wounds demonstrated to induce faster wound closure rates in the beginning than controls in groups with higher pulse rates
\end{abstract}

and frequencies of the shock waves. Furthermore, the regeneration of microcirculation and perfusion in the healing skin was significantly improved after the application of, in particular, higher pulse rates as given by increased numbers of perfused capillaries and functional vessel density. The study of inflammation showed, especially in high-pulse ESW groups, higher leukocyte counts, and rolling leukocytes over time until day 6 as a response to the induction of inflammatory reaction after ESW application. Angiogenesis showed a marked increase in positive areas as given by sprouts, coils, and recruitments in all ESW groups, especially between days 4 and 6. Conclusion: The major findings of this trial demonstrate that ESW therapy to skin wounds is effective and safe. This is demonstrated by the initially faster wound closure rate, but later the same wound closure rate in the treatment groups than in controls. Furthermore, during the regeneration of microcirculation and perfusion in the healing skin, a significant improvement was observed after the application of, in particular, higher ESW pulse rates, suggesting an ESWrelated increase in nutrient and oxygen supply in the wound tissue.

(c) 2021 S. Karger AG, Basel karger@karger.com www.karger.com/esr (c) 2021 S. Karger AG, Base
Correspondence to:

Heiko Sorg, heiko.sorg@t-online.de 


\section{Introduction}

Wound healing is a physiological process which, through the formation of a new tissue, causes the repair of the injury and thus the closure of a wound. A large number of cells and factors are responsible for this process, which can be negatively influenced in many places [1]. If these factors are not eliminated, this can lead to a disturbance of wound healing with the subsequent development of a chronic wound [2]. The wound healing of complicated or chronic wounds represents a severe impairment of the quality of life for every patient affected [3]. In addition, the treatment of chronic wounds is an enormous burden on any health care system in terms of loss of resources and enormous costs [4]. It is therefore imperative to find cost-effective, noninvasive, and effective treatment options that will accelerate and complete the healing of problem wounds and reduce the associated treatment costs.

Extracorporeal shock waves (ESWs) have been used in medicine for almost 30 years now. The treatment of sports injuries, pseudarthrosis, and orthopedic soft tissue pathologies are recognized treatment indications [5]. In recent years, ESW therapy (ESWT) has shown some successful results in the field of wound healing $[5,6]$. The indication areas range from the treatment of diabetic or infected wounds to large-area wounds in burn medicine and the improvement of healing of flaps in plastic reconstructive surgery. Thus, Haupt and coworkers were the first to report in detail on the positive effect of ESW on wound healing in an animal model in 1990. The working group was able to demonstrate a significantly reduced duration of wound closure in Yorkshire pigs [7]. Based on the very positive results of clinical studies [8], research on the effects of shock waves on wound healing, then again, became increasingly interesting. Numerous clinical studies followed. In animal experiments on rats, a significantly longer survival time of the epigastric abdominal skin flap with reduction of the necrosis zones was shown after treatment with ESW [9]. After the first positive results from these animal studies, the first human patients with impaired wound healing were finally treated with ESW. In 2007, the results of a feasibility study from Austria were published, in which 208 patients with nonhealing complicated chronic wounds were treated with shock waves. The indications ranged from posttraumatic impaired wound healing to arterial and venous ulcers, pressure sores, and burn wounds. Complete epithelialization was achieved in up to $75 \%$ of patients treated [10]. A further study was able to achieve similarly good results and confirmed the positive effects of ESW on wound healing [11]. In the following, the group around Schaden and colleagues could further demonstrate in a presentation of midterm results that wound closure appeared in nearly $75 \%$ after 2 ESW treatments and did not reappear at the treated locations again. Interestingly, comorbidities had no significant influence on this effect [12]. In a meta-analysis, ESW on acute and soft tissue wounds could further demonstrate to be of better therapeutic effects than standard wound therapy alone; however, the authors recommended further trials with a higher quality for better evidence [6]. All these indications and studies have in common, however, that the underlying mechanism of action has only been partially deciphered to date. In particular, the state of knowledge regarding the effect of ESW on microcirculation and vascularization is poor. Moreover, the methods are often not studied in a standardized way. In this context, we were therefore able to establish the Diver Box model in advance, which enables the standardized investigation of ESWs in experimental wound healing research [13]. In the present work, we will attempt to gain new pathophysiological insights into the effect of ESW on microcirculation and angiogenesis using an animal model.

\section{Methods}

Implantation of the Dorsal Skin Fold Chamber and Wounding A total of 40 female BALB/c mice (Charles River, Sulzfeld, Germany; $12-15$ weeks old) with a body weight of 18-22 g was used for the study. The animals were housed in standard laboratories with a 12-h light-dark cycle, and had free access to standard laboratory food (R/M-H, $10 \mathrm{~mm}$, ssniff Spezialdiäten $\mathrm{GmbH}$, Soest, Germany) and water ad libitum. The experiments were conducted in accordance with guidelines for the Care and Use of Laboratory Animals and the Institutional Animal Care and Use Committee (Ruhr University of Bochum, Medical Faculty, Bochum, Germany, no.: 8.87-50.10.37.09.135). The experiments were started after a 1 -week acclimatization period of the animals. The dorsal skin fold chamber in mice was used for intravital microscopy, as previously described by our group [13-16].

\section{ESW Application}

For the standardized application of ESW, mice are transferred to the Diver box as previously described (Fig. 1) [13]. The shock wave was applied in deep analgosedation to the animals. The animals of the control group were subjected to the same procedure with analgosedation and immersion in the water bath. For the ESW induction, the electrohydraulic method was used (Dermagold, MTS Europe GmbH, Konstanz, Germany). In accordance with the literature, only shock waves with an energy flux density of $0.1 \mathrm{~mJ} / \mathrm{mm}^{2}$ were used in our experiment. The shock waves were applied with 500 or 1,000 pulses per application with an application duration of $20 \mathrm{~s}$ with a defocusing transducer $[5,17,18]$. 


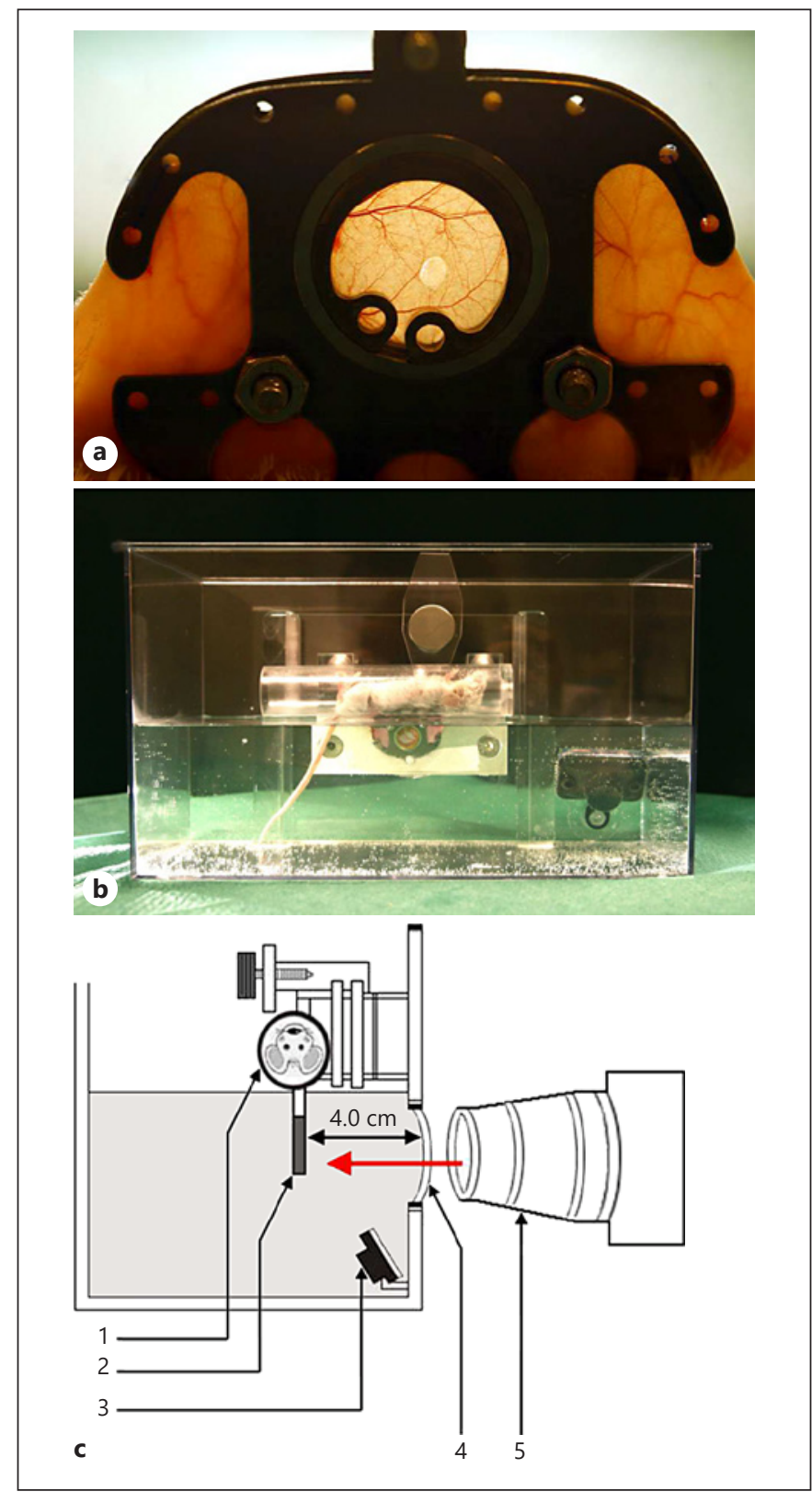

Fig. 1. a The dorsal skin fold chamber after implantation in a $\mathrm{BALB} / \mathrm{c}$ mouse with the standardized skin wound is shown. Through the observation window, the skin muscle with vessels and the central transparent wound surface can be seen with the view of the opposite dermis. $\mathbf{b}$ The mouse positioned in the Diver Box with the implanted dorsal skin chamber, which is completely surrounded by water. c The schematic shock wave application in the Diver Box. The mouse (1) can be seen in the holder with the implanted dorsal skinfold chamber (2) immersed in the tempered water bath of the Diver Box, which is equipped with a temperature measuring device with indicator (3), an application window with coupling membrane (4) through which a shock wave applicator (5) can emit the shock waves (red arrow). The device ensures a standardized distance between the applicator and the wound area of $4.0 \mathrm{~cm}$ (double arrow). Modified from [13].

\section{Study Design}

Mice were divided into 3 therapy groups $(n=30)$ and one control group $(n=10)$. The 3 therapy groups were treated with shock waves at different pulse rates and application frequencies. The energy density of $0.1 \mathrm{~mJ} / \mathrm{mm}^{2}$ remained constant for all 3 therapy groups. Photographic documentation and intravital microscopic examination were carried out on a day-by-day basis in all animals on the following days: day 1 ( $24 \mathrm{~h}$ after surgery), day 2 (72 h after surgery), day 4 (120 h after surgery), and day 6 (168 $\mathrm{h}$ after surgery). The experimental groups were divided as follows: (group 1) first ESW application with 500 pulses/min on day 0, second ESW application with 500 pulses/min on day 6; (group 2) ESW application with 1.000 pulses/min on day 0; and (group 3) first ESW application with 1.000 pulses/min on day 0 , second ESW application with 1.000 pulses/min on day 6 . In the control group, no ESW application was performed; however, the animals of the control group were subjected to the same treatment conditions and application procedures as the therapy groups to ensure comparability, that is, they were anesthetized and immersed in the water bath.

\section{Analysis of Wound Healing}

All procedures were performed in anesthetized mice. Analysis of wound healing was performed using the photomacroscopic setting of a camera (EOS D60, Canon; Krefeld, Germany) using planimetric techniques (Optimas 6.2; Meyer Instruments, Inc., Houston, TX, USA). Wound closure was considered complete when the entire surface area was epithelialized (Fig. 2). Values are given as relative decrease of wound area in relation to the initial value.

\section{Microscopy and Microcirculatory Analysis}

Intravital microscopy and microcirculatory analysis have been performed as described by our group previously $[14,19,20]$. Microscopic images were taken and recorded by a video system for off-line evaluation via a charge-coupled device video camera (AVT-BC 71, AVT-Horn, Aalen, Germany) and monitored on a television screen (PVM-1453 MD, HR Trinitron Color Video Monitor, Sony, Köln, Germany). An analog-to-digital converter (MovieBox DV ${ }^{\circledR}$, Pinnacle Systems, Inc., Mountain View, CA, USA) was connected to the video camera for video recording and storage. This was connected with a computer (Acer Extensa 5620$1 \mathrm{~A} 1 \mathrm{G} 12$, Germany) and with an external USB hard disk for archiving. The program Pinnacle Studio ${ }^{\text {TM }}$ HD Ultimate 15 (Avid Technology GmbH, Munich) was used to edit the videos. In addition to the quantitative evaluation of the microcirculation by the parameters listed above, a parameter was defined which should provide both quantitative and qualitative information about the blood vessel formation actually occurring in vivo along the wound margin of the intramuscular lesion. This was designated as angiogenesis-positive area (APA). In order to visualize and differentiate the different angiogenesis mechanisms, the following criteria were defined for the presence of an APA: (1) sprouts, morphological changes of a blood vessel such as blood vessel buds or shoots are a criterion for the angiogenesis positivity in a healing tissue and indicate that classical angiogenesis (sprouting); (2) coils and capillaries, characterized by a worming of the vascular wall, were defined as a sign of one of the mechanisms of nonsprouting angiogenesis: vascular stretching and enlargement (remodeling and pruning); and (3) recruitment, existing nonperfused vascular beds were examined, from which new vessels were formed by reperfu- 


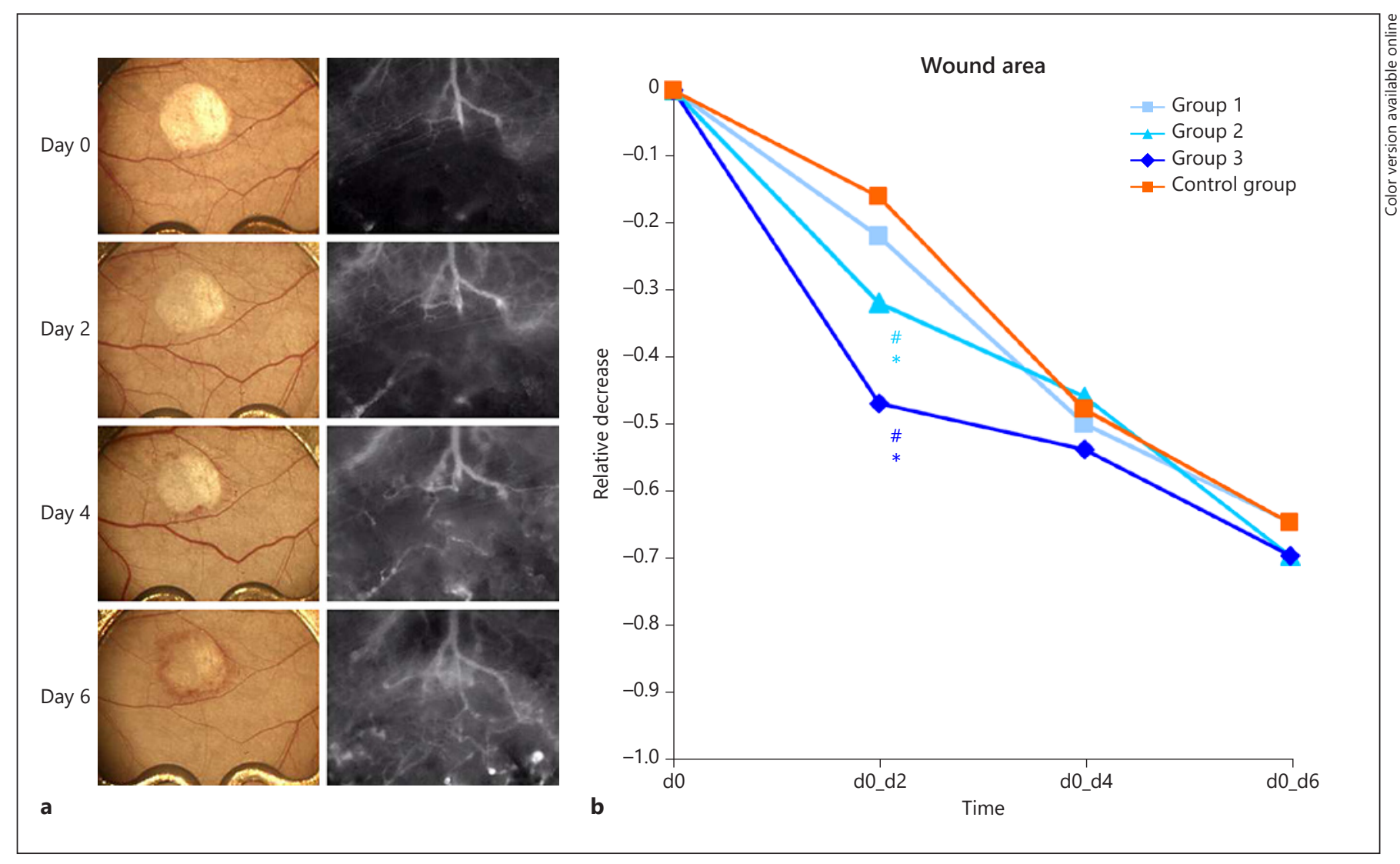

Fig. 2. a The left panel shows the photographic documentation of the skin fold chamber window in the time course of day 0 to day 6 with corresponding intravital fluorescence microscopic images $\times 10$ magnification showing the edge of the wound on the right panel. b The relative decrease of skin wound closure in relation to the initial value is demonstrated. Data series up to d6 for groups $1-3$ and control. ${ }^{*} p<0.05$ versus group $1,{ }^{*} p<0.05$ versus the control group. sion. The investigation and counting of the APA were carried out manually and were indicated in number per analysis field.

\section{Statistical Analysis}

All data are given as mean. Data were analyzed for normality and equal variance across groups. As no normality for the here presented results could be detected, differences between groups were assessed using one-way ANOVA by the Kruskal-Wallis comparison followed by the appropriate post hoc comparison test (Wilcoxon-Mann-Whitney). The overall statistical significance was set at $p<0.05$. Statistics were performed using the software package Stata 11.2 SE (StataCorp LLC, College Station, TX, USA).

\section{Results}

\section{Diver Box and ESW Application}

Using the newly developed Diver Box, shock waves could be applied in vivo without mechanical tissue damage. Even with multiple applications and a higher pulse rate, no higher complication rates than in the control group were observed, so that good clinical tolerability of the shock wave application in the Diver Box could be assumed [13].

\section{Wound Healing}

Planimetric analysis of the wounds showed a constant decrease of the wound area in relation to the initial value for all examination groups from day 0 . In all groups, a decrease in the wound area of an average of $0.65 \mathrm{~mm}^{2}$ at d6 in relation to day 0 could be observed. Significant decreases compared to controls as well as group 1 occurred only in groups 2 and 3 at day 2 (Fig. 2). From day 2, the wound area is constantly reduced, and no relevant differences could be detected between the groups (Fig. 2).

\section{Microcirculatory Analysis}

Intravital microscopic analysis revealed a marked increase in vessel diameters over the study time of 6 days; 


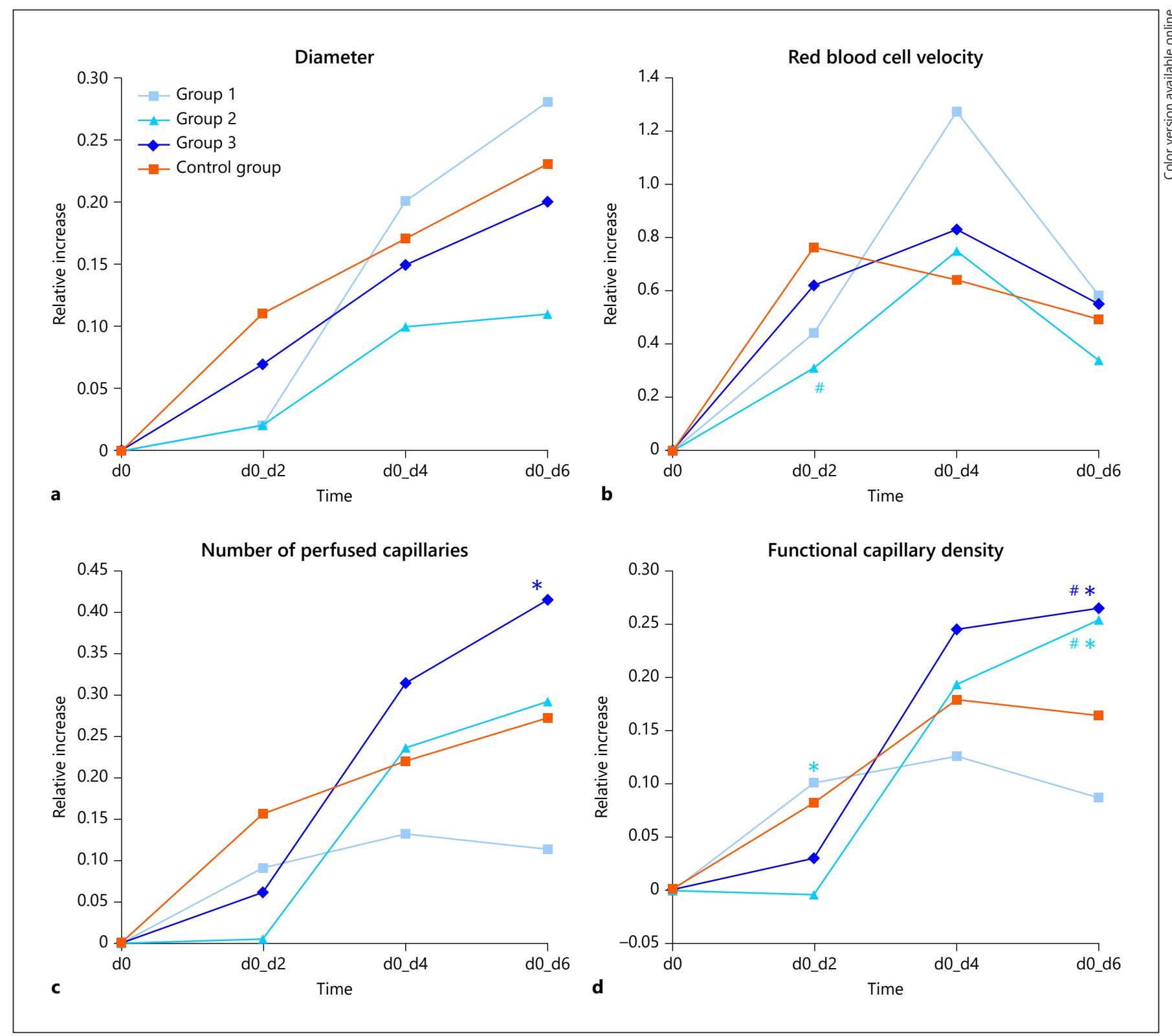

Fig. 3. Microcirculatory analysis with the measurement of the vessel diameters (a), red blood cell velocity (b), NPC (c), and functional capillary density $(\mathbf{d}) .{ }^{*} p<0.05$ between ESW groups, ${ }^{*} p<$

0.05 versus the control group. ESW, extracorporeal shock wave; NPCs, number of perfused capillaries.

however, with no statistically significant difference (Fig. 3a). A rapid increase in the RBCV can be observed in all groups (Fig. 3b). By day 4, the RBCV tends to increase further, but then decreases in all groups, however, without significant differences over time (Fig. 3b). The number of perfused capillaries (NPCs) increased continuously in all groups during the time course of the examination until day 6. Only the repeated application of

1,000 pulses/min (group 3) showed a significantly higher NPC on day 6 compared to the group with only a single administration of 500 pulses/min on day 0 (group 1; Fig. 3c). The control group showed no statistically significant differences over time among groups. Functional capillary density showed a continuous increase over time in all groups (Fig. 3d). Interestingly, in group 2, almost no growth could be observed in the ratio of day 2 to the 


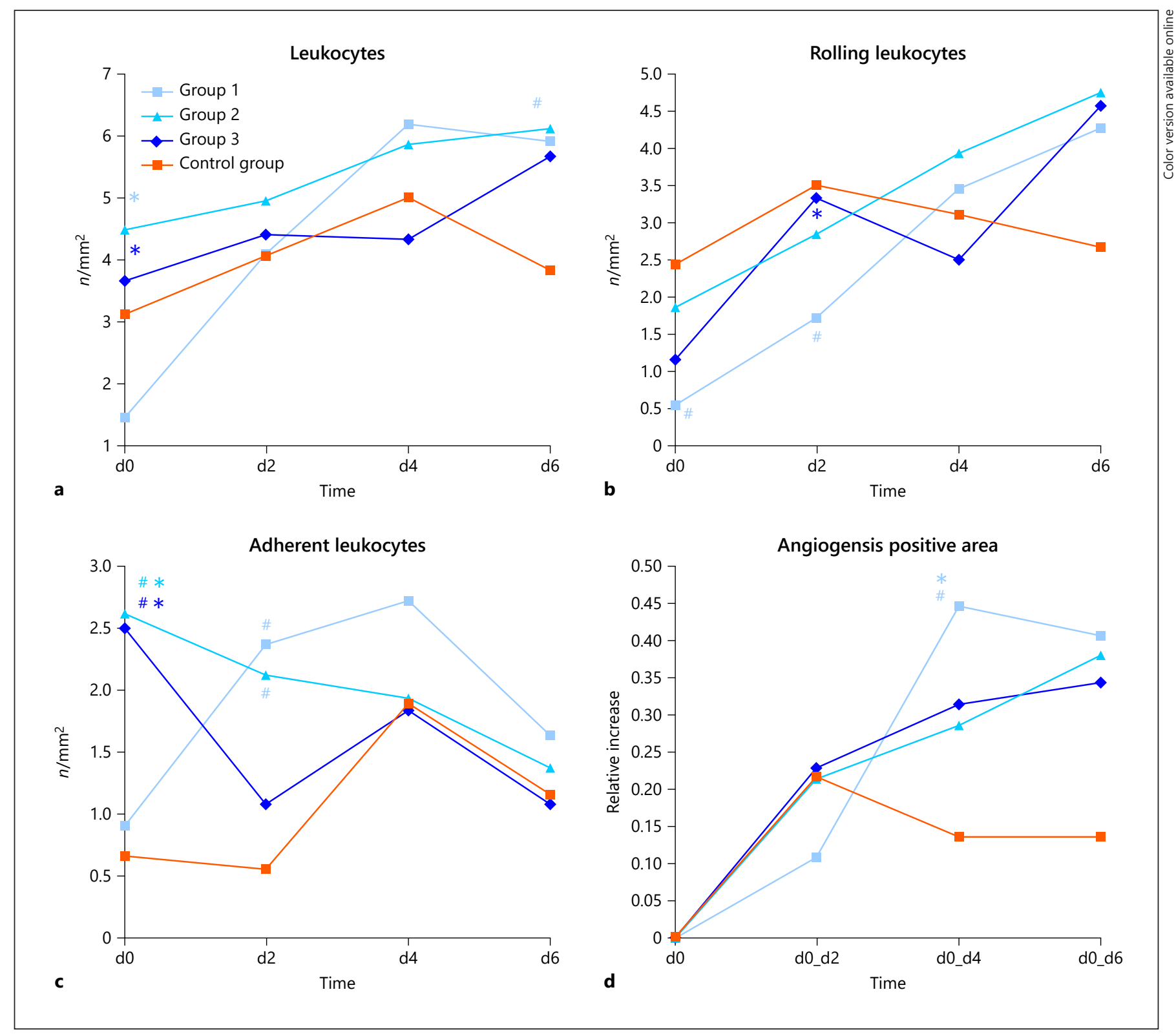

Fig. 4. Inflammation and angiogenesis study with the number of leukocytes (mean values): number of rolling leukocytes (a); number of adherent leukocytes (b); and the number of APA (c); on all

examination days (d0-d6) for groups $1-3$ and control (d). ${ }^{*} p<$ 0.05 between ESW groups, ${ }^{\#} p<0.05$ versus the control group. ESW, extracorporeal shock wave; APA, angiogenesis-positive area.

initial value at day 0 , while group 1 only showed the highest increase in FCD up to day 2, with a relatively flat increase at day 4 , followed by a significant regression at day 6 in comparison to the other groups ( $p<0.05$ vs. groups 2 and 3 at d6). Notably, groups 2 and 3 with the higher doses of ESW showed only slower increases in FCD at the beginning of the application up to day 2, however, increased then with highest FCD values at day 4 and sig-

nificantly higher FCD values at day 6 among groups (Fig. 3d).

\section{Inflammation and Angiogenesis Study}

Leukocyte counts increased among all groups starting with the lowest count at day 0 in group 1 and with significantly highest values in groups 2 and 3 (Fig. 4a). Over time, leukocyte counts further increased in all ESW groups 
resulting in a markedly lowest count in the control group at day 6 in comparison to all other groups (groups 1-3) as a response to the induction of inflammatory reaction after ESW application. Approximately, the same can be seen in the evaluation of rolling and adherent leukocytes (Fig. 4b, c). While the control group with the significantly highest number of rolling leukocytes starts at d0, day 6 shows the lowest number compared to the ESW groups. In the evaluation of the adherent leukocytes, the high-dose ESW groups start with significantly higher values of adherent leukocytes compared to the group 1 and control group, but then all drop to the same level on day 6 .

Angiogenic evaluation showed a marked increase of APA up to day 2 with lowest values in group 1 . From day 2, the groups showed different developments (Fig. 4d). Groups 2 and 3 showed a largely parallel continuous upward trend and reached their maximum at $\mathrm{d} 6$. The control group fell back to the lowest APA values of all groups from day 2 and remained at this value until day 6 . Interestingly, in group 1, the maximum APA value was registered on day 4 and was thus above all other group values, in particular, significantly higher than the values of the control group and groups 2 and 3.

\section{Discussion}

ESWs have been shown to have a positive effect on wound healing [5]. In the present study, their effect on microcirculation and angiogenesis was examined by using the Diver Box model for the standardized application of ESWs and intravital fluorescence microscopy in an animal model. The major findings of this trial demonstrate that ESWT to skin wounds is effective and safe [13]. This is demonstrated by the initially faster wound closure rate, but later the same wound closure rate in the treatment groups than in controls. Furthermore, in the investigation of the regeneration of microcirculation and perfusion in the healing skin, a significant improvement was observed after the application of, in particular, higher pulse rates, suggesting an ESW-related increase in nutrient and oxygen supply in the wound tissue. These findings should be discussed in detail. In the area calculation of the wound, all groups showed a constant decrease in relation to the initial value from the first day of examination. A significantly strong relative decrease compared to the control group was observed in shock wave groups 2 and 3 (single and double application of 1,000 pulses). This significance can only be determined relatively early at the beginning of the experimental period from day 0 to day
2. This significantly accelerated wound area reduction indicates a favorable influence of shock waves on early wound healing and suggests that the effectiveness of ESW is dependent on the pulse rate, however, with no differences at later time points.

When considering NPC and FCD as the functional indicators of tissue perfusion, our results show the general regeneration of microcirculation in all groups over the time course of the examination. First of all, this allows the conclusion that a damage via the application of ESW can largely be ruled out in the context of skin wound healing. Furthermore, the NPC and FCD increase in all groups in relation to the baseline value. Since the baseline value was measured only after the wound was set, an increase in vessel density was expected to reflect perfusion recovery in all groups. Overall, groups 2 and 3, with higher and repetitive ESW application of 1,000 pulses, showed the highest FCD values in comparison to the lower pulse rate as well as to the control group. This reflects that higher ESW pulses lead to increased epithelization in the beginning of skin wound healing, which is followed by a significantly increased regeneration of microvascular architecture.

The acute inflammatory reaction is characterized by the immigration of leukocytes into the affected wound area [21]. In the presence of trauma, the leukocytes released from the bone marrow are recruited into the inflammatory area by inflammatory mediators. There, a leukocyte-endothelial interaction takes place, which finally enables the leukocytes circulating in the bloodstream to adhere to the vessel wall and then to migrate from the vessel wall into the inflammatory tissue. In our study, the total number as well as the number of rolling leukocytes as a sign of inflammation after wound healing increases in all groups over the course of time, while the number of adherent leukocytes in the groups until day 4 is very different, and settles at a very similar level on day 6. The inflammatory effect of ESW has already been controversially discussed in previous experiments. On the one hand, there are studies that describe an anti-inflammatory effect especially on macrophages [22-24]. On the other hand, Calcagni and colleagues [25] found evidence for a slight proinflammation by shock wave treatment. This observation has already been confirmed by studies of ESW application in burn wound models as well [26, 27]. Our data seem to support the finding that shock waves have a rather pro-inflammatory effect, which in this context can be seen as a necessary inflammatory reaction to induce and accelerate angiogenesis in the wound healing process. It is known that, in particular, polymorphonuclear neutrophil granulocytes express various an- 

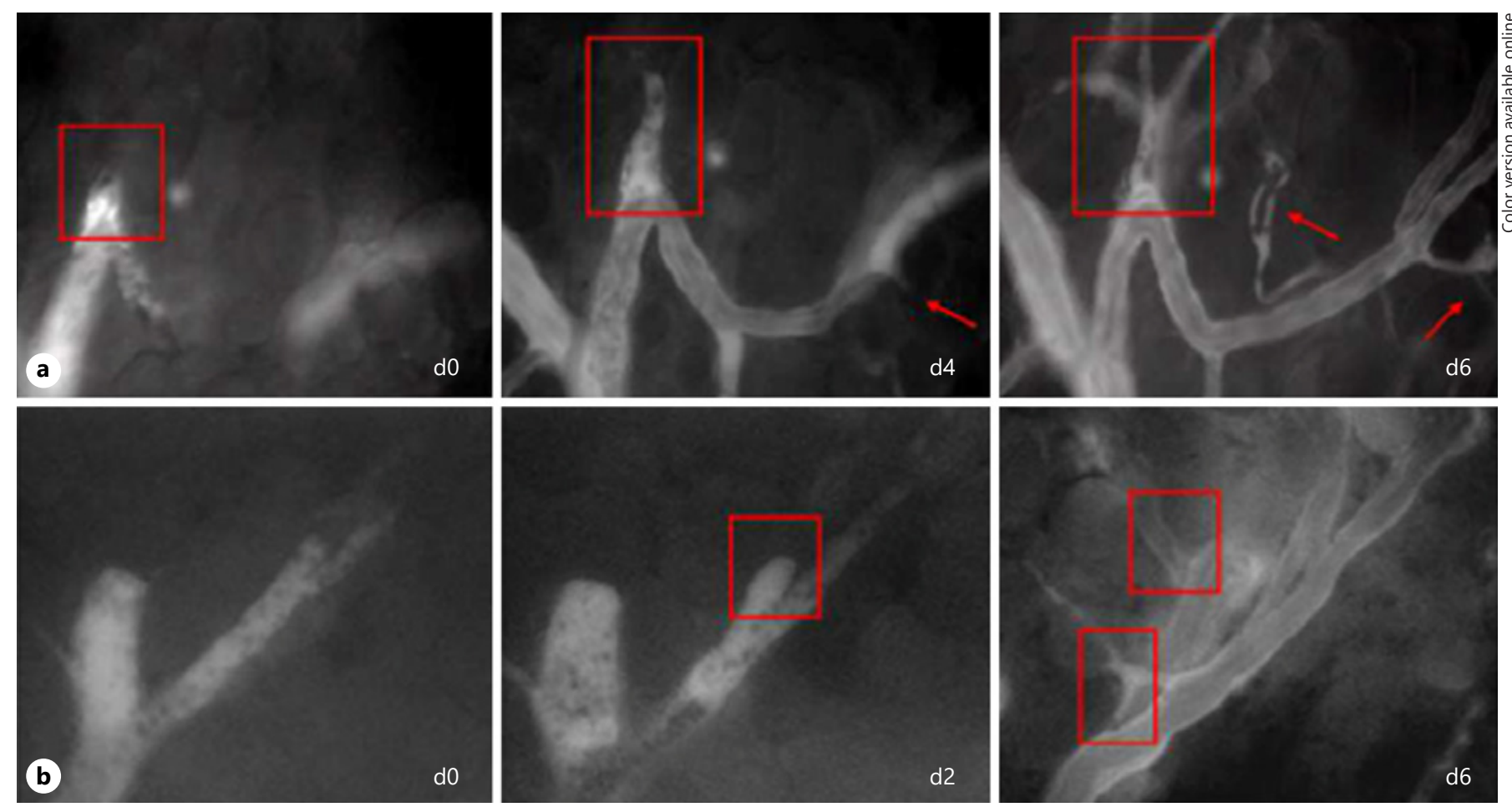

Fig. 5. Intravital fluorescence microscopic images in $\times 20$ magnification of the angiogenesis in the wound area of a mouse dorsal skin fold chamber. a Several blood vessel sprouts can be seen. On day d0, 2 vascular stumps can be seen at first, which are not connected to each other. In the left of the 2 vessels, even thrombotic blood cells can be seen as signs of blood stasis (dark recesses). On day d4, a connection of the 2 vessels with a clear blood flow can be detect- ed. In addition, the bud of a new vessel (red square, $\mathrm{d} 4$ ) is formed from the vessel stump by proliferation of the endothelial cells. On day d6, this bud splits into several capillaries (red square, d6). In addition, angiogenesis can be seen, which has also developed from $\mathrm{d} 4$ to $\mathrm{d} 6$ to new capillaries (red arrows in $\mathrm{d} 4$ and $\mathrm{d} 6$ ). b An area of sprouting of an arteriole in the period from day d0 to d6. giogenesis-relevant factors and have a proangiogenic effect. Even the adhesion of polymorphonuclear neutrophil granulocytes to the activated endothelium before migration into the inflammatory tissue mediates the synthesis of angiogenesis-relevant factors such as TNF-alpha, IL-1, IL-6, and IL-8 in the endothelial cells. Furthermore, leukocytes store and release VEGF in the granules [28] and is considered one of the most important factors in the induction of angiogenesis [29].

Since the main focus of this study has been the investigation of ESW effects on wound healing and angiogenesis, the consideration of leukocyte-endothelium interaction in APA was of central importance. The fact that ESW have an effect on angiogenesis has already been investigated using a variety of different models $[5,11,17,26,30-32]$. In a similar experimental model other than the presented study, however in a healthy skin tissue, it could be concluded that shock waves have a proangiogenic and pro-inflammatory effect [25]. In our study, however, ESW were applied to traumatized, wounded skin, which induces an inflammatory reaction per se. Inflammatory angiogenesis is the basic prerequisite for successful wound healing in the context of an inflammatory process because the blood vessels that enter the wound not only ensure the increased nutrient and oxygen demand in the wound tissue but are also characterized by a high vascular permeability, which allows plasma components such as fibrinogen to escape. The fibrin produced by extravasal coagulation forms the matrix for the immigration and proliferation of tissue cells during wound healing [33]. The evaluation of APA using fluorescence microscopy provided both quantitative and qualitative information about the in vivo angiogenesis. The first newly built vessels were already visible on day 2 (Fig. $5 \mathrm{a}$ ). The different angiogenesis mechanisms such as sprouts, coils, and recruitment of vessels could be visualized. In addition to sprouts, which could be detected in the capillary network as well as in third- and fourth-order arterioles, numerous tortuous formations of the vessel walls and unperfused oc- 
cluded vessels were visible, which were perfused again in the course of the procedure (Fig. 5b). Thus, 2 different mechanisms could be observed in our study, which cause the increase of neovascularization: I - the recruitment of existing, initially unperfused microvasculature, which reopens its flow path; and II - the actual formation of new blood vessels, mainly by previous tortuosity and subsequent budding from a preformed capillary system. In the quantitative evaluation of the APA, the control group fell back to a minimum, compared to all other groups from day 2 , and remained at this level until the end of the observation. In accordance with the leukocyte values, the control group plays an only subordinate role in the evaluation of APA. These results indicate shock wave-induced intensified angiogenesis. The curves of the APA, however, do not show a clear correlation to the FCD, which is surprising at first, since both parameters are indicators for neovascularization and angiogenesis processes. However, the lack of correlation can easily be explained by the fact that APAs indicate how many new capillaries are formed, even if they consist of still tiny buds and sprouts, which do not significantly influence the FCD. The FCD, on the other hand, measures the length of the capillaries; it is calculated by dividing the total length of the measured capillaries by the measuring area of the chamber window, so the values of the APA can increase without increasing the FCD. However, the number of APA does not correlate with the NPC either because the number of existing capillaries can decrease as capillaries close or are reabsorbed by remodeling processes if they are damaged at the wound margin, and the number of APAs increases at the same time because new vessels are formed from the wound margin. Thus, the counting of APA quantified mechanisms of angiogenesis that were not covered by other parameters such as the wound area calculation, capillary count, or functional capillary density.

Taken together, ESW application showed to be of great benefit to the skin wound healing process such as the epithelialization and the microcirculatory regeneration. This encourages the use of ESW application as an additive therapy to standard wound therapy. This was also confirmed in a recently published meta-analysis, but further studies should be conducted to be able to use ESWT in acute or chronic wounds as evidence-based medicine [6].

\section{Acknowledgement}

The authors thank Mr. Christian Dorfmüller (Dermagold, MTS Europe GmbH, Konstanz, Germany) for technical support of the study.

\section{Statement of Ethics}

The experiments were conducted in accordance with guidelines for the Care and Use of Laboratory Animals and the Institutional Animal Care and Use Committee (Ruhr University of Bochum, Medical Faculty, Bochum, Germany, no.: 8.87-50.10.37.09.135).

\section{Conflict of Interest Statement}

The authors declare no conflicts of interest.

\section{Funding Sources}

This research study was supported by the German Social Accident Insurance (DGUV) (project no.: FF-FR 0160).

\section{Author Contributions}

H.S. participated in study planning, data collection, and analysis and interpretation of the results and wrote the manuscript. I.Z. performed the animal experiments and analyzed the data. D.J.T. participated in the supervision of the research and correcting the manuscript. J.K. participated in data analysis, interpretation of the results, and correcting the manuscript. J.H. participated in supervision of the research and correcting the manuscript. O.G. participated in data analysis and interpretation of the results. N.S. participated in data analysis, interpretation of the results, and correcting the manuscript. S.L. participated in supervision of the research and correcting the manuscript. A.R. participated in the study conception and interpretation of the results and was a major contributor in writing the manuscript. All authors read and approved the final manuscript.

\section{References}

1 Rodrigues M, Kosaric N, Bonham CA, Gurtner GC. Wound healing: a cellular perspective. Physiol Rev. 2019 Jan 1;99(1):665-706.

2 Eming SA, Martin P, Tomic-Canic M. Wound repair and regeneration: mechanisms, signaling, and translation. Sci Transl Med. 2014 Dec 3;6(265):265sr6.

3 Kapp S, Miller C, Santamaria N. The quality of life of people who have chronic wounds and who self-treat. J Clin Nurs. 2018 Jan; 27(1-2):182-92.

4 Chien CW, Brown T, McDonald R, Brown T, McDonald R, McDonald R. Examining content validity and reliability of the assessment of children's hand skills (ACHS): a preliminary study. Am J Occup Ther. 2010;64(5): 756-67.

5 Mittermayr R, Antonic V, Hartinger J, Kaufmann H, Redl H, Téot L, et al. Extracorporeal shock wave therapy (ESWT) for wound healing: technology, mechanisms, and clinical efficacy. Wound Repair Regen. 2012; 20(S6):456-65.
Sorg et al. 
6 Zhang L, Fu XB, Chen S, Zhao ZB, Schmitz C, Weng CS. Efficacy and safety of extracorporeal shock wave therapy for acute and chronic soft tissue wounds: a systematic review and meta-analysis. Int Wound J. 2018 Aug;15(4): 590-9.

7 Haupt G, Chvapil M. Effect of shock waves on the healing of partial-thickness wounds in piglets. J Surg Res. 1990;49(1):45-8.

8 Fukumoto Y, Ito A, Uwatoku T, Matoba T, Kishi T, Tanaka H, et al. Extracorporeal cardiac shock wave therapy ameliorates myocardial ischemia in patients with severe coronary artery disease. Coron Artery Dis. 2006 Feb; 17(1):63-70.

9 Meirer R, Brunner A, Deibl M, Oehlbauer M, Piza-Katzer H, Kamelger FS. Shock wave therapy reduces necrotic flap zones and induces VEGF expression in animal epigastric skin flap model. J Reconstr Microsurg. 2007 May;23(4):231-6.

10 Schaden W, Thiele R, Kölpl C, Pusch M, Nissan A, Attinger CE, et al. Shock wave therapy for acute and chronic soft tissue wounds: a Feasibility Study. J Surg Res. 2007;143(1):112.

11 Saggini R, Figus A, Troccola A, Cocco V, Saggini A, Scuderi N. Extracorporeal shock wave therapy for management of chronic ulcers in the lower extremities. Ultrasound Med Biol. 2008 Aug;34(8):1261-71.

12 Wolff KS, Wibmer A, Pusch M, Prusa AM, Pretterklieber M, Teufelsbauer $\mathrm{H}$, et al. The influence of comorbidities and etiologies on the success of extracorporeal shock wave therapy for chronic soft tissue wounds: midterm results. Ultrasound Med Biol. 2011 Jul;37(7): 1111-9.

13 Sorg H, Tilkorn DJ, Kolbenschlag J, Zwetzich I, Hauser J, Goertz O, et al. A novel technique for the standardized application of shock waves in experimental research: the diver box. Ultrasound Med Biol. 2018 Jul;44(7):1563-8.

14 Sorg H, Krueger C, Vollmar B. Intravital insights in skin wound healing using the mouse dorsal skin fold chamber. J Anat. 2007;211(6): $810-8$.
15 Sorg H, Krueger C, Schulz T, Menger MD, Schmitz F, Vollmar B. Effects of erythropoietin in skin wound healing are dose related. FASEB J. 2009;23(9):3049-58.

16 Ring A, Langer S, Tilkorn D, Goertz O, Henrich L, Stricker I, et al. Induction of angiogenesis and neovascularization in adjacent tissue of plasma-collagen-coated silicone implants. Eplasty. 2010;10.

17 Stojadinovic A, Elster EA, Anam K, Tadaki D, Amare M, Zins S, et al. Angiogenic response to extracorporeal shock wave treatment in murine skin isografts. Angiogenesis. 2008; 11(4):369-80.

18 Qureshi AA, Ross KM, Ogawa R, Orgill DP. Shock wave therapy in wound healing. Plast Reconstr Surg. 2011;128(6):721e-7e.

19 Ring A, Goertz O, Al-Benna S, Ottomann C, Langer S, Steinstraesser L, et al. Accelerated angiogenic induction and vascular integration in a novel synthetic scaffolding matrix for tissue replacement. Int J Artif Organs. 2010 Dec;33(12):877-84.

20 Langer S, Beescho C, Ring A, Dorfmann O, Steinau HU, Spindler N. A new in vivo model using a dorsal skinfold chamber to investigate microcirculation and angiogenesis in diabetic wounds. GMS Interdiscip Plast Reconstr Surg DGPW. 2016;5:Doc09.

21 Nathan C. Points of control in inflammation. Nature. 2002 Dec 19-26;420(6917):846-52.

22 Mariotto S, de Prati AC, Cavalieri E, Amelio E, Marlinghaus E, Suzuki H. Extracorporeal shock wave therapy in inflammatory diseases: molecular mechanism that triggers anti-inflammatory action. Curr Med Chem. 2009; 16(19):2366-72.

23 Davis TA, Anam K, Amare M, Naik S, Peoples GE, Tadaki D, et al. Extracorporeal shock wave therapy suppresses the early proinflammatory immune response to a severe cutaneous burn injury. Int Wound J. 2009;6:11-21.
24 Sukubo NG, Tibalt E, Respizzi S, Locati M, d'Agostino MC. Effect of shock waves on macrophages: a possible role in tissue regeneration and remodeling. Int J Surg. 2015 Dec; 24(Pt B):124-30.

25 Calcagni M, Chen F, Högger DC, Lindenblatt N, Keel M, Giovanoli P, et al. Microvascular response to shock wave application in striated skin muscle. J Surg Res. 2011;171(1):347-54.

26 Goertz O, Lauer $H$, Hirsch $T$, Ring A, Lehnhardt M, Langer S, et al. Extracorporeal shock waves improve angiogenesis after full thickness burn. Burns. 2012;38(7):1010-8.

27 Goertz O, Hauser J, Hirsch T, von der Lohe L, Kolbenschlag J, Stricker I, et al. Short-term effects of extracorporeal shock waves on microcirculation. J Surg Res. 2015 Mar;194(1):30411.

28 George D, Yancopoulos SD, Gale NW, Rudge JS, Wiegand SJ, Holash J. Vascular-specific growth factors and blood vessel formation. Nature. 2000;407:242-8.

29 Risau W. Mechanisms of angiogenesis. Nature. 1997 Apr 17;386(6626):671-4.

30 Kuo YR, Wang CT, Wang FS, Chiang YC, Wang CJ. Extracorporeal shock-wave therapy enhanced wound healing via increasing topical blood perfusion and tissue regeneration in a rat model of STZ-induced diabetes. Wound Repair Regen. 2009;17(4):522-30.

$31 \mathrm{Ha}$ CH, Kim S, Chung J, An SH, Kwon K. Extracorporeal shock wave stimulates expression of the angiogenic genes via mechanosensory complex in endothelial cells: mimetic effect of fluid shear stress in endothelial cells. Int J Cardiol. 2013 Oct 9;168(4):4168-77.

32 Goertz O, von der Lohe L, Lauer H, Khosrawipour T, Ring A, Daigeler A, et al. Repetitive extracorporeal shock wave applications are superior in inducing angiogenesis after full thickness burn compared to single application. Burns. 2014;40(7):1365-74.

33 Wong ME, Hollinger JO, Pinero GJ. Integrated processes responsible for soft tissue healing. Oral Surg Oral Med Oral Pathol Oral Radiol Endod. 1996 Nov;82(5):475-92. 